\title{
DIRECT-WRITE SELF-ASSEMBLY OF 3D COLLOIDAL MICROSTRUCTURES
}

\author{
Justin Beroz, Mostafa Bedewy, and A. John Hart \\ Mechanosynthesis Group, Department of Mechanical Engineering \\ University of Michigan, Ann Arbor, Michigan, USA
}

\begin{abstract}
We present a direct-write technique for assembly of microscale 3D colloidal crystals on substrates. We use a custombuilt high-resolution liquid manipulation system to dispense colloidal suspensions through a capillary tip. Using this system, we establish a liquid bridge between the capillary tip and a temperature-controlled substrate, initiating crystal growth upward from the substrate. We demonstrate construction of cone-shaped and tower structures by controlling the dynamic shape of the liquid meniscus during crystal precipitation. Interplay between lateral capillary forces and granular cohesion governs assembly. Finally, we show that confinement of the meniscus on microfabricated template features enables assembly of discrete particle clusters.
\end{abstract}

\section{INTRODUCTION}

Spanning from the nanometer to millimeter scales, discrete components can serve as building blocks for optical components, filters, biosensors, micromechanisms, electronics, and many other novel materials and devices. New techniques are needed to assemble a growing library of these building blocks with high precision and throughput, ranging from semiconductor nanoparticles to polymer microspheres and miniature circuit elements. In particular, the proportional relationship between liquid meniscus curvature and capillary pressure is a versatile means for ordering ensembles of particles at liquid-air interfaces, and enables fabrication of closely packed layers and discrete clusters of micro and nanoparticles on substrates. Collectively, these techniques are known as evaporative self-assembly, and are, for example, implemented by dispensing droplets onto the target substrate by inkjet printing [1], or by drawing a meniscus across the substrate [2]. However, it is highly challenging to arrange particles into well-organized 3D shapes by current methods. Here, we demonstrate that $3 \mathrm{D}$ colloidal assemblies can be directly fabricated on flat and microstructured surfaces by dynamic mechanical control of a liquid meniscus during evaporation.
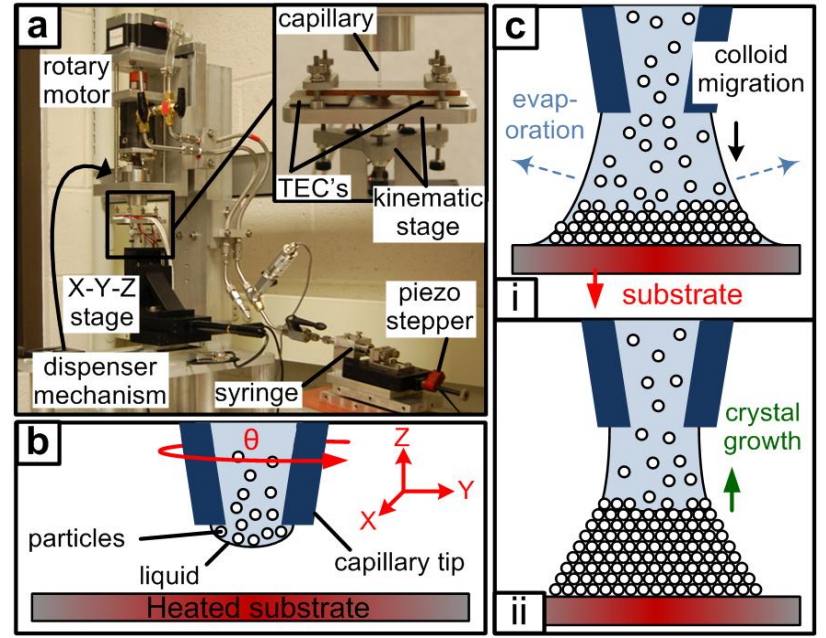

Figure 1: (a) Liquid dispensing system (adapted from [3]); (b) schematic of 4-DOF motion control, and (c) schematic of directwrite assembly process.
To facilitate this study, we designed and built a precision instrument (Fig. 1a, [3]), which can simultaneously deposit and manipulate nano- and microliter liquid droplets on a temperature controlled substrate, in four degrees of freedom (Fig. 1b). In this paper, we introduce a technique for fabricating 3D colloidal microstructures using vertical stage motion. We contact the heated substrate with a liquid that is displaced slightly from the end of the capillary tip, thereby creating a liquid bridge between the capillary tip and substrate (Fig. 1c). Particles in the liquid migrate towards the substrate due to sedimentation and/or evaporation-driven liquid flow. When the evaporation rate locally exceeds the rate of liquid replenishment, the meniscus recedes upward from the substrate, building a colloidal crystal whose geometry is determined by the dynamic shape of the receding meniscus.

Via concerted control of vertical substrate motion and liquid dispensing rate, we can control the liquid bridge profile between the tip and the heated substrate. As shown in Figure 2, this control allows us to fabricate colloid crystals, consisting of $10 \mathrm{~m}$ polystyrene particles, in the shape of cones and towers on flat substrates.

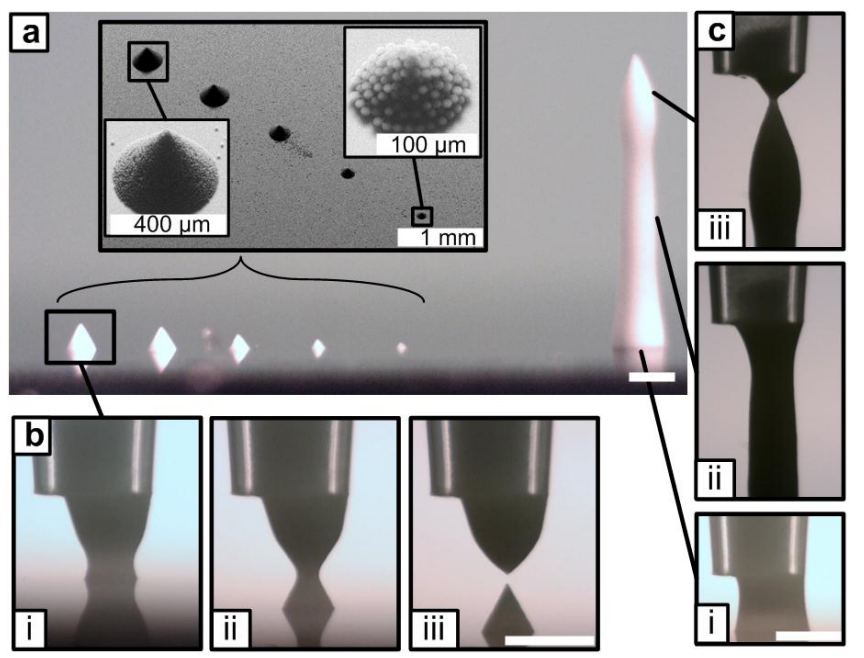

Figure 2: Extrusion of crystals of $10 \mathrm{~m}$ polystyrene spheres into conical and tower structures by drawing from a capillary tip (all scale bars $=500 \mathrm{~m}$ unless noted)

\section{PROCESS FUNDAMENTALS}

In order to understand how these structures precipitate from the liquid bridge, we present a conceptual description of the particle assembly mechanisms observed during crystal growth, which arise due to capillary forces. Specifically, the local curvature of a liquid meniscus is proportional to the pressure difference across the interface, as described by the Laplace-Young equation. This capillary pressure, in concert with the effects of surface tension, produce two distinct phenomena which we call surface assembly and bulk cohesion. Formation of these crystal structures may be understood by considering the interaction between colloid organization at the liquid-air interface and coherence in the bulk material. 


\section{Assembly at the Three-Phase Contact Line}

Suspended colloid particles that are at the liquid-air interface locally deform the liquid meniscus, which gives rise to lateral capillary attraction between particles due to interface tension and Laplace capillary pressure. This drives the colloids to assemble into closed-packed domains along the liquid-air interface. This has been explored thoroughly in the context of self-assembly of closed-packed monolayers and multilayers of particles on surfaces, using a variety of techniques, including [4] drop-casting, bladecasting, and Langmuir-Blodgett drawing. After evaporation, particles remain arranged in the crystal lattice due to Van der Waals attraction to neighboring particles and to the surface.

Our process may be regarded as a 3-dimensional (3D) analog to these well established planar self-assembly techniques. However, here are important differences with regard to our process due to the 3D contour of the liquid bridge. As shown in Fig. 3, evaporation-induced flows pull particles towards the liquidsubstrate contact line, where the particles pack due to lateral capillary forces at the interface, thus causing a colloid crystal nucleate and to grow. In this respect, the colloid microstructure may be thought of as precipitating from the meniscus-crystal contact line in the direction of meniscus recession.

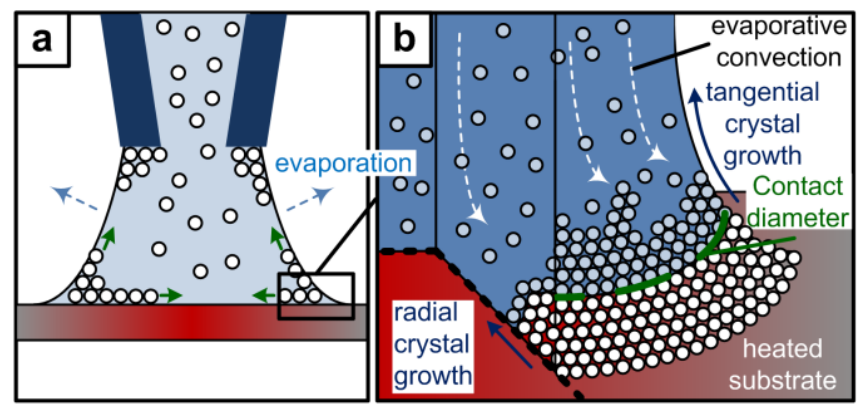

Figure 3: (a) Schematic illustrating the surface assembly process within the liquid bridge; (b) isometric view with wedge cut illustrates crystal growth tangent to the meniscus, and inwards radial crystal growth, which adds thickness to the crystal.

Meniscus recession may occur tangent to the meniscus surface, thereby precipitating a closed-packed outer surface; and radially inwards, thereby thickening the closed packed surface to form a multi-layered 'crust' (Fig. 3). Note that evaporationinduced convection can transport particles to the meniscus surface for tangential crystal growth, and to the underside of the hexagonal-packed surface for radial crystal growth.

Several factors influence the integrity of the closed packed crystal. One obvious culprit is monodispersity in colloid diameter. Larger-than-average particles in the crystal lattice create defects that can propagate through the crystal. This may be minimized by using highly monodisperse particles. Additionally, crystal growth may nucleate at multiple locations, which leads to grain boundaries at the intersection of crystals with different lattice orientations. These defect mechanisms are well documented in the literature for 2D capillary self-assembly [4]. Geometry provides a unique constraint for our process. The crystallization process is confined by the curvature of the meniscus, and defects may be created to accommodate this geometric confinement. One can imagine that the defect density will increase radially inwards from the outer surface, ultimately resulting in jammed, amorphously packed colloids at the center.

\section{Bulk Cohesion}

In contrast to the surface assembly just described, capillary forces can effectuate bulk cohesion between particles. Here, the colloid particles behave as an amorphously packed wet granular material, which may exist in four distinct phases according to the volume fraction of liquid in the void spaces between the particles [5]. At very low saturation, small liquid bridges form between particles near contact points (Fig 4a-i). Surface tension and capillary pressure within the liquid bridges constitute a normal capillary force attraction, which causes high cohesion amongst the particles.

Increase in liquid content fills more of the void space between the particles, which leads to coalescence of capillary bridges (Fig. 4a-ii) and a decrease in particle cohesion. At the limit where all void space is filled with liquid (Fig. 4a-iii), the particles experience weak cohesion due to lateral capillary forces at the peripheral liquid air interface. Further increase in liquid content dilutes particles into a slurry (Fig. 4a-iv), and cohesion is lost.

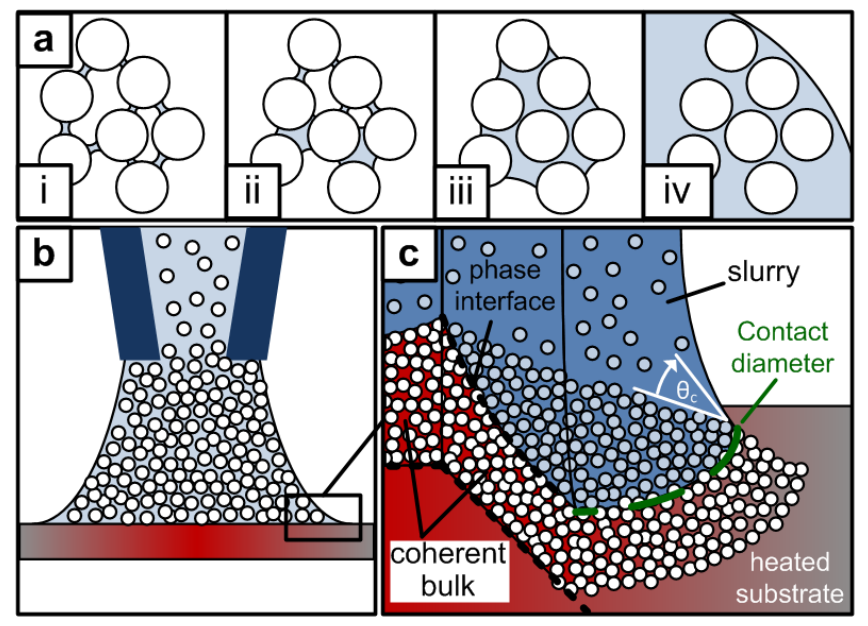

Figure 4: (a) Schematic illustrating the phases of granular bulk cohesion [5]; (b) illustration of particles sedimenting within the liquid bridge; (c) isometric view with wedge cut illustrates the contact angle, $\theta_{c}$, created at the contact diameter at the interface between the cohesive bulk phase and liquid bridge slurry.

During extrusion of a crystal structure by our method, particles may sediment onto the substrate inside the liquid bridge (Fig. 4b). Substrate heating accelerates liquid evaporation, thereby introducing air voids in the interstitial spaces and effectuating cohesion amongst the sedimented particles (i.e., phases $i$ and ii, Fig. 4a). Collectively, the particles behave as a distinct bulk wet granular material that, we believe, develops a discernable interface with respect to the liquid bridge slurry above (Fig. 4c). The meniscus of the liquid bridge slurry pins to the cohesive bulk at the peripheral contact diameter with contact angle $\theta_{c}$, as illustrated. An amorphous crystal structure may be grown by simply pulling on the meniscus so that this contact diameter recedes by slipping over the cohesive bulk material.

\section{RESULTS}

Based on this understanding, we demonstrate control of the process parameters to direct the crystal structure contour, and realize $3 \mathrm{D}$ structures, ranging from cones to towers. Utilizing the capabilities of our liquid dispenser system, we are able to control the following parameters for fabricating colloid microstructures: profile of the liquid bridge between the capillary tip and 
precipitating crystal structure, initial particle concentration, and substrate patterning. In the following section, we demonstrate, experimentally, the possibilities within this design space and enumerate the interplay between surface assembly and bulk cohesion in guiding 3D colloid assembly.

\section{Contact Diameter (i.e., slope) Control}

During the assembly process, the meniscus of the liquid bridge extending from the capillary tip is locally pinned to the crystal structure at the contact diameter, as illustrated in Fig. 3 and Fig. 4. Due to contact angle hysteresis, this angle may vary between characteristic advancing and receding contact angles.

By controlling the rate of liquid dispensing and the downward speed of the substrate, we can control this contact angle (within the advancing-receding range). This, in turn, enables us to control the evolution of the contact diameter with time, and this determines the slope of the particle assembly. Qualitatively speaking, larger contact angles (i.e., near the characteristic advancing contact angle) increase the contact diameter over the course of crystal growth, and smaller contact angles (i.e., near the characteristic receding contact angle) decrease the contact diameter. Two limits of this technique are conical structures (Fig. 2a,b), where we constrict this precipitation diameter at a constant rate during crystal growth; and towers (Fig. 2a,c), where we maintain a constant contact diameter and can extrude a vertical column of indefinite length.

To better understand the mechanics of assembly, we fabricated an array of conical crystals, where we systematically varied the initial contact diameter, substrate motion rate, and liquid volume fraction in the tip. In all experiments, we held the substrate temperature at $70^{\circ} \mathrm{C}$, and did not dispense additional liquid once the bridge was formed. Under these control parameters, we fabricated cones ranging in height from $50 \mathrm{~m}$ to $550 \mathrm{~m}$, and having a wide range of height-to-base diameter aspect ratios (0.41.2). Notably, we observed various slope contours, such as those depicted in Fig. 5a,b.

In order to interpret these results, we studied the evolution of the liquid meniscus over the course of crystal growth. Fig. 5a,b illustrates the evolution of the liquid bridge meniscus profile over time by overlaying images captured at periodic time intervals (Fig. $5 \mathrm{a}=10 \mathrm{sec}$, Fig. $5 \mathrm{~b}=5 \mathrm{sec}$ ). For each instant, we measured the contact angle of the liquid meniscus with respect to slope of the final structure, and identified three distinct stages, which are labeled (i), (ii), and (iii) in Fig. 5a,b.

(i) Upon establishing a liquid bridge, the meniscus is initially pinned to the substrate at a relatively large contact angle. Withdrawing the substrate stretches the meniscus, thereby decreasing the contact angle until it reaches the characteristic receding angle with respect to the structure slope, which we measure to be $\sim 11-15^{\circ}$.

(ii) As crystal growth proceeds, the meniscus contact line recedes at this constant angle. Notably, this contact angle is the same in Fig. 5a and Fig. 5b (within measurement error), even though the structure contours are significantly different. We believe this indicates that bulk cohesion is present within the volume, and the meniscus is slipping over the cohesive material interface at a characteristic receding contact angle, which we would expect to be independent of the interface contour. Our hypothesis is supported by observation of amorphous packing at the crystal surface in this region, as shown by the SEM images (Fig. 5c,d).

(iii) Towards the apex of the cone, we observe an increase in measured contact angle accompanied by a change in slope. Here, we propose that the liquid content in the bridge has lowered to the point where the entire liquid bridge becomes a coherent granular material. At this point, although we state a meniscus contact angle in the contour plots (Fig. 5a,b), formally calling this a contact angle is no longer appropriate. A more accurate interpretation is that we have a coherent wet granular column, which necks under the applied tension until rupture occurs.

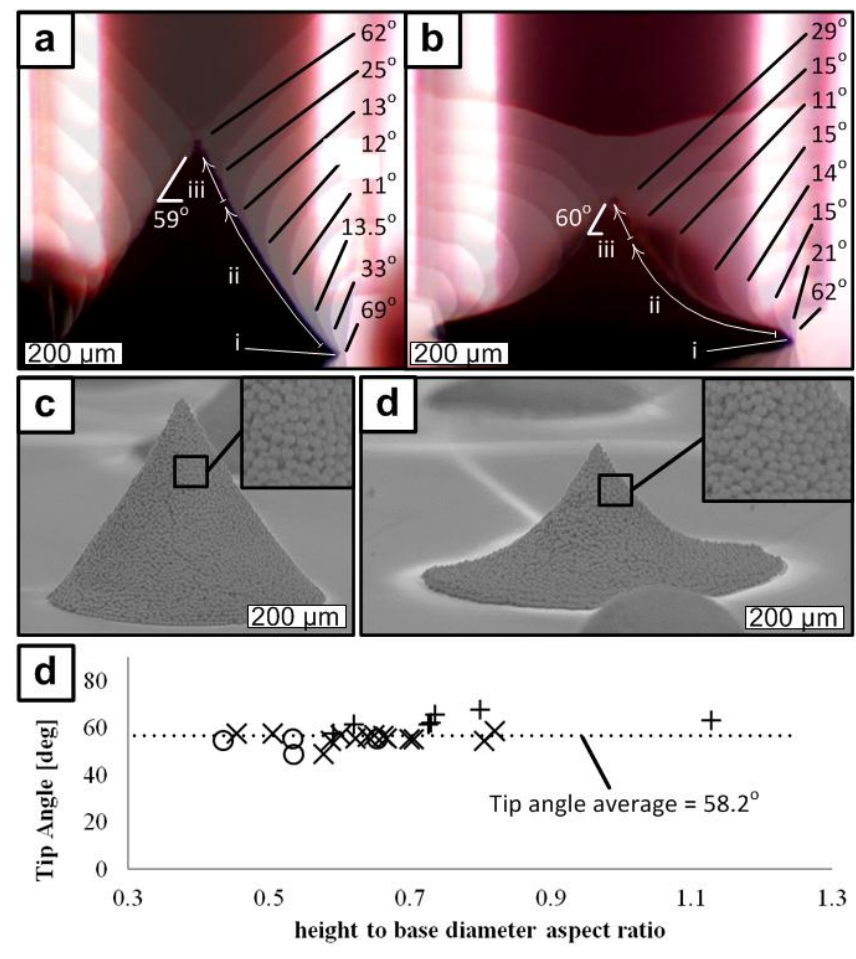

Figure 5: $(a, b)$ contour plots demonstrating the evolution of the meniscus during crystal growth; (c, d) SEM images of these structures reveal amorphous crystal packing; (d) the slopes for all cones converge to $\sim 60^{\circ}$ near the apex.

A further interesting observation is that the apex slope is approximately the same for all the cones we fabricated in this study, even though the initial slopes vary. The plot of cone apex angle vs. aspect ratio (Fig. 5d) shows that this angle appears to be independent of aspect ratio and draw speed. This seems to indicate a characteristic yielding behavior for this cohesive wet granular material.

\section{Crystallinity Control}

For surface assembly to occur, the suspended particles must have enough mobility to travel to, and arrange at, the liquid air interface. Particle cohesion and physical obstruction can terminate surface assembly by arresting free particles from maneuvering to the liquid-air interface, or limiting the space available to arrange in a closed-packed lattice, respectively.

In our technique, sedimentation of particles is substantial, and thus growth of the bulk cohesive phase through these sedimented particles is responsible for terminating the radial and tangential growth of surface assembled crystals. By controlling the initial concentration of particles at bridge formation, we can prescribe how much time free particles have to assemble at the surface before termination by the growing coherent bulk phase.

Figure 6 shows three different broken particle towers fabricated with different initial particle concentrations at liquid bridge formation. Note that these 'initial concentrations' are 
qualitative. We controlled this by simply allowing different amounts of time for particle sedimentation in the capillary tip before creating the liquid bridge with the substrate. The tower with the highest initial particle concentration (Fig. 6a) exhibits an entirely amorphous outer surface and inner volume. Here, the initial particle concentration is high enough that there is already coherence among the particles, so surface assembly is completely arrested. At lower initial particle concentration (Fig. 6b), we achieved a polycrystalline surface approximately 1 particle thick. At our lowest prepared particle concentration (Fig. 6c), we achieved a hexagonal closed packed outer surface on the tower. This closed packed layer was able to grow radially inwards a thickness of several particle layers before becoming jammed by the amorphous bulk phase.

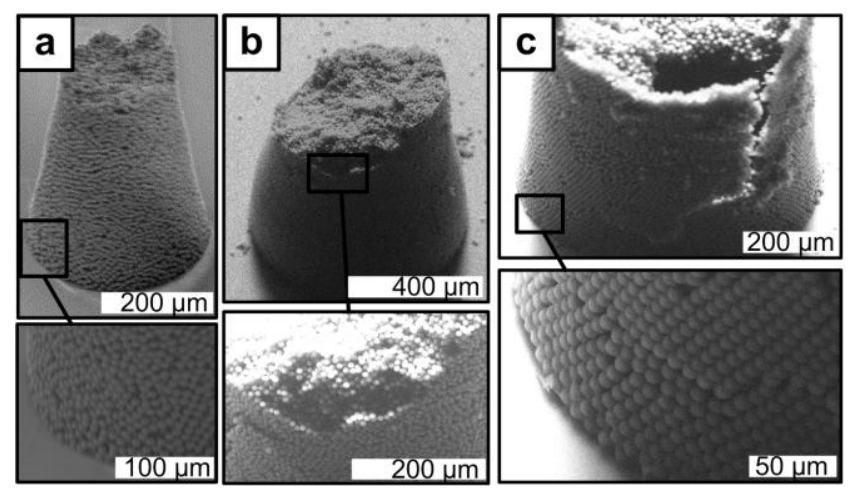

Figure 6: Three broken towers, fabricated from liquid bridges having different initial particle concentration (decreasing left to right) each reveal differences in crystal packing at the surface.

\section{Discrete Particle Assemblies}

In the results presented so far, the base diameter of the crystal structure is limited by the size of the capillary tip and the geometry of the liquid bridge. Here, we show that pinning the meniscus to a substrate microfeature allows fabrication of highly ordered assemblies comprising discrete numbers of particles. Notably, these structures are substantially smaller than the tip diameter, and their placement is determined by registry with the guide feature. This enables precision deterministic fabrication of colloid crystal structures with dimensions and placement precision finer than the capillary tip dimensions and substrate motion resolution, respectively.

To demonstrate this principle, we chose to assemble particles onto vertically aligned carbon nanotube (CNT) microstructures. As shown in Fig. 7a, we assembled a small crystal arrangement of particles across the opening of a CNT hollow micro cylinder (50 $\mathrm{m} \mathrm{ID}, 80 \mathrm{~m}$ OD). Notice that the meniscus can easily adapt to misalignment between the capillary tube axis and CNT microcylinder without adversely affecting particle placement accuracy. Similarly, we deposited clusters of two, three, and four particles on top of densified CNT micropillars (Fig. 7b,c,d respectively). For these results, the liquid (water) wicked through the CNT pillar, causing it to densify due to capillary forces. Thus, we are demonstrating the potential for using the suspension liquid to affect the organization on a surface by other capillary-driven mechanisms in concert with particle crystal growth.

This result also demonstrates the potential of this technique to deterministically place individual, and discrete arrangements of, microparticles by engineering microfeatures having suitable geometry and surface energy. Our technique can potentially be scaled down to smaller sizes. One can imagine, for instance, depositing a single $500 \mathrm{~nm}$ particle on a $1 \mathrm{~m}$ diameter post.

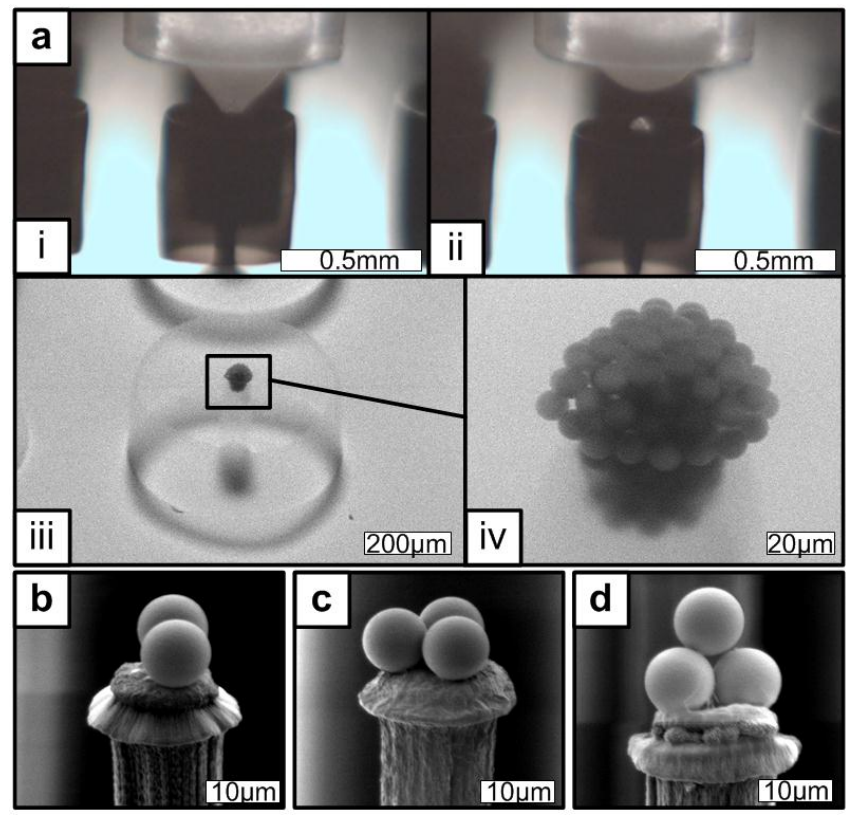

Figure 7: The colloid suspension meniscus can be pinned to diameters smaller than the capillary tip diameter by substrate microfeatures, such as (a) concentric carbon nanotube (CNT) microcylinders and solid CNT micropillars in order to deposit discrete arrangements of particles such as a (b) dimer, (c) trimer, and (d) tetramer.

\section{CONCLUSION}

Our approach to direct-write evaporative self-assembly contrasts conventional approaches to inkjet printing [6] and electrohydrodynamic printing $[7,8]$, which are limited by the size, ejection rate, and spread of droplets on the substrate. We showed that, by maintaining direct mechanical control of the meniscus, it is possible to directly build a complex 3D shape or to direct individual particles towards guide features on the substrate. This is potentially advantageous for heterogeneous assembly and integration of pre-made components such as microelectronic elements on circuit boards or MEMS devices. Deterministic colloid assemblies may be useful as miniature filters or photonic crystals within lab-on-a-chip systems, or model granular materials having engineerable thermal, electrical, or mechanical properties. Additionally, this technique can potentially be scaled down to assemble colloidal nanoparticles by using smaller diameter tips. This may be interesting for direct fabrication of novel photonic crystal arrays and other metamaterials.

\section{REFERENCES}

[1] Calvert, P., Chem. Mat., 2001. 13(10): p. 3299-3305.

[2] Bodnarchuk, M., et. al., ACS Nano, 2010. 4(1): p. 423-431.

[3] Beroz, J., et al., Rev. Sci. Instrum., 2012. 83: 015104.

[4] Galisteo-Lopez, J.F., et al., Adv. Mater., 2011. 23: p. 30-69.

[5] Mitarai, N., Nori, F., Adv. in Physics, 2006. 55(1): p. 1-45.

[6] Derby, B., Ann. Rev. Mater. Res., 2010. 40: p. 395-414.

[7] Mishra, S., et al., J. Micromech. Microeng., 2010. 20(9).

[8] Park, J.U., et al., Nano Letters, 2010. 10(2): p. 584-591.

\section{CONTACT}

*A.J. Hart, tel: +1-734-615-6146; ajohnh@umich.edu 